\title{
Virtual Phase Dynamics for Constrained Geometries in a Soap Froth
}

\author{
Yu Feng, Heather J. Ruskin and Bao Zhu \\ School of Computer Appl ications, Dubl in Ci ty Uniersity, Dubl in 9, I reland \\ Corresponding emai l: hruskin@compapp. dcu.ie
}

\begin{abstract}
Soap froths as typical disordered ellular structures, exhibiting s patial and t empoal evolution, have been s tudied t hrough $t$ heir distributions and topological properties. Recently, persistence has been i ntroduced as a non-topological probe to study froth dynamis at different length s cales and to vi ew the froth as a two-phase system. Using a di rect simul ation method, we have i nvestigated vi rtual phase dynamis in $2 \mathrm{D}$ artificial froths with var ious initial structures corresponding to controlled disorder. In particular, we exami ne the special case of a dfect ring s urrounding a central inclusion i n a unform froth, for different percentages of persistent c ells, where this ge ontery permi ts compaison with shelltheory. It appears that defect location and pattern of cell inclusion in the virtual phase cause considerable variation in the evolutionary Heaviour, leading to non- universal exponents for the phase dynami cs. This is probably explained by the fact that the froth is still inhe transient pe riod over simul ation tme-scales, rather than achieving the final stage of persistence. However, distinctive patterns of $r$ esponse can be identified $f$ or the di fferent froth egions, despite the limi tations on \$stem size.
\end{abstract}

Ke ywords persistence, defect ring, phase dynamics, constrained geometries, transience

\section{Introduction}

Soap froth as a space-filling cellular network $\mathrm{B}$ an intrinsically non-equilibrium system, which has attracted considerable interest in recent years, [1-4]. The froth system evolves to a universal stable state through surface-energy driven diffusion, by means of topological rearrangements in the bubbles (cells). In twodimensions, these may occur in two ways, namely through side- $s$ witching and cell disappearance, (T1 and T2 processes respectively).

The area of an individual cell changes wi th tme during di ffusion, according to von Neumann's Law, [5], wher e cel ls wi th number of ides, $n<6$ will shrink, whilst those wth $n>6$ will grow. Typi cally, dynami c poperties ar e measured in terms of the side distribution, $f(n)$ and i ts second moment, $\mu_{2}$, whh achieves a stable val ue afer a certain period of time, i.e. reaches a scaling state. Nonequilibrium behaviour has also been studied, and transient effects have been shown to depend on the nat ure and amount of disorder in the or iginal structure. In particular, introduction of one or more defects (i.e. topological dislocations) 
in an otherwise uniform hexagonal froth affects the speed at which scaling is reached, while a constant $\mu_{2}$ is not obtained in the special case of a single defect, [6-9].

Additionally, topological order between neighbouring cells has been described by correlation laws, such as that of Aboav-Weaire, [10-12], although more recent studies have indicated that second and third nearest-neighbours are also correlated, i.e. that theories of froth dynamics based on independent bubble approximations are inadequate, [13]. These detailed correlations are based on analysis of the froth as a system of concentric shells, with layers, $j=1,2 \ldots n$ arranged around a central germ cell, $j=0$. The froth is said to have the property of shellstructured inflatability if no topological inclusions occur between layers, [14], but most $S S I$ froths do not retain the property under system evolution, [15].

While topological measures are a natural choice to describe froth, more general measures can provide a useful basis for comparison with other non-cellular structures. The local decay of persistence, $P\left(t_{0}, t\right) \sim t^{-\theta}$ was first proposed as a new and general probe of non-equilibrium dynamics, [16-18]. Although agreement between theory and experiment on the value of $\theta$ is lacking, treating the froth as a two-phase system, (with a virtual phase defined by colouring a fraction of the bubbles), permits exploration of the dynamics at different length scales.

Persistence exponents for volume fractions $0 \leq \phi \leq 1$ in a coarsening $2 \mathrm{D}$ froth have been investigated both experimentally and computationally, [16-17,19], but no evidence was found for persistence decay in a Voronoi system over simulation time-scales. This, in contrast to experiment, where findings were obtained over extended times. However, for a uniform froth, seeded with one or more defects, some indications of persistent decay have been obtained, [19], although it was not found possible to estimate exponent values with any confidence. Nevertheless, this has suggested that controlling the rate of evolution may be possible for specified geometries in order to observe further persistence effects or, at least, to quantify the dynamics of the virtual phase. In what follows, the focus is principally on the evolution of the virtual phase in a uniform froth, where the geometry observed consists of a ring of defects around a central defect. The methodology is described and results on the dynamics and persistent properties are reported.

\section{Methodology}

A ring geometry was chosen since, given that only a small percentage of the original cells will contribute to persistence effects, rapid cell suppression is initially required, coupled with the potential for large bubbles to grow. Further, unlimited growth is checked by the ring buffer, so that the froth is not consumed, while the equidistant spacing provides some basis for scaling the observed effects on the froth partition, e.g. [8] and not unlike [20].

The direct simulation method [21-22] employed, provides precise information on individual cell and topological changes as these occur. A distinction is made between topological and diffusive changes and detailed statistics on cell pressure, 
area, number of sides etc. are recorded at each time step. Periodic boundary conditions are chosen and equilibration takes place through an iterative procedure. After local relaxation, the froth is analysed for T1/T2 changes. Unlike an intrinsically disordered froth, the uniform froth is stable, (or in mechanical equilibrium), so that evolution must be stimulated. This is achieved by forcing a T1 or T2-formed process, to create a topological dislocation or defect. Clearly, defects are "survivors" (since $n>6$ ), and account for a large percentage of the remaining area as the froth evolves [23]. Further, if defects form part of the virtual phase, they will dominate persistent behaviour.

The virtual phase, (Figs. 1 and 2), is defined by colouring a given fraction $\phi$ of the bubbles at time $t_{0}$ and studying persistent properties of this fraction as $t \rightarrow t_{f}$. The number of survivors is crucial as an indicator of the potential for further change. At any time $t$, quantities of interest include $\left\langle A^{*}(t) / A(0)>\right.$ and $N^{*}(t) / N(t)$, (namely, the average persistent area ratio, where $A(0)$ is the initial bubble area, and the fraction of coloured bubbles persisting (or persistent region)). The ring geometry network consists in general of a number of defects equi-distant from a single central defect and equi-spaced in relation to each other. (We focus here predominantly on a 12-defect ring, but also consider one with 8 defects). Different choices of cells, for inclusion in the virtual phase of the ring geometry, are illustrated by the concentric shadings around the central defect in Fig. 1. These correspond to volume fraction, $\phi=0.2,0.4,0.6$ respectively (where $\phi=0.4$ includes the inner ring for $\phi=0.2$ and $\phi=0.6$ includes both inner rings). Taking the radius as defined by the number of cell layers crossed from the centre cell to the ring formed by the defects, several ring sizes have also been investigated. Fig. 2 shows the evolution of the basic geometry for given $\phi$, where all defects increase in area over time and finally impact upon each other, (Fig. 2 (c)). Systems of size up to 2500 bubbles have been considered for the special ring geometry case, forming a basis for comparison with random and other colouring patterns for the virtual phase dynamics, [19].

\section{Results}

The evolutionary picture for the ring geometry shows the froth partitioned into the central defect, inner ring (compressed by defects expanding from both the centre and defect ring), defect "buffer" zone and the external region, where none of the defects form part of the (shaded) virtual phase. On impact of the defects in the ring, no further evolution is possible and a constrained equilibrium is restored. The behaviour of the overall second moment $\mu_{2}$ vs $t$ for the ring geometry is similar to that for the single defect case initially, with higher peaks (as several defects suppress smaller bubbles), but with some indication that this rapid rise slows down as defects compete, (for $\mu_{2}$ around 1.4). Fig. 3 contrasts the ring behaviour with that of a random (Voronoi) froth and the single defect case. Similarly, average bubble area $\langle A(t)\rangle$ vs $t$ is consistent with the single defect initially, but again diverges slightly, at $t \sim 150$ time steps, as coarsening slows down, (not shown). Large area bubbles are thus still relatively few in number 


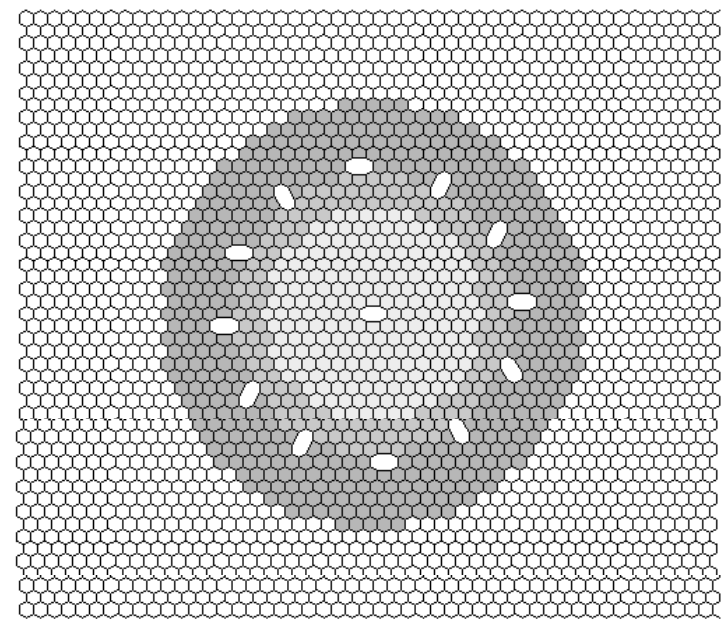

Fig. 1. Hexagonal froth (system size $=2500$ ) with ring defects, with shaded area represen ting coloured vlume fraction $\phi=0.04,0.2,0.4$.

in the froth as a whole. In terms of persistence, the average area of persistert regions within a bubble at time $t$ normalised w.r.t. that at $t_{0}$, (average area ratio), and the fraction of bubbles containing persistent volume at time $t$ w ere also considered and are shown in Figs. 4 and 5 respectively for small and large radius ring sizes, $\left(r_{s}=11, r_{l}=15\right)$ and for $\phi$ values in the range 0.06-0.4.

In Fig. $4,<A^{*}(t) / A(0)>$ vs $t$, persistent area declines gradually, since the bubbles forming the virtual phase are being squeezed by the central defect and buffer ring. This is observed for both the small and large ring, with the steepest decline in each case corresponding to the value of $\phi$ for which the virtual phase just exceeds the inner region, (i.e. $\phi=0.2,0.4$ for the small and large ring respectively). This is in broad agreement with the results obtained for the single and several defects cases [19], and little difference is shown for colouring one or a few defects of ring.

F or the persistent fraction of bubbles, $\log N^{*}(t) / N(t)$ vs. $\log \langle A(t)\rangle$ in Fig. 5 shows broadly similar results to those discussed in [19], with the steepest slope again observed for the virtual phase having volume fraction $\phi$ corresponding closely to the ring radius. It is very evident that the slope $k$ vs $\phi$ is highly variable, (comparing also with the case of one and several defects, [19]), and that there is a rapid decline in persistent fraction for some $\phi$ values, (Fig. 6). Specifically, for those where the radius of the virtual phase is close to or just larger than the defect ring radius. The effect is most marked where the coloured ring has small radius, (see e.g. Fig. $1, \phi=0.04$ say), since the coloured bubbles 

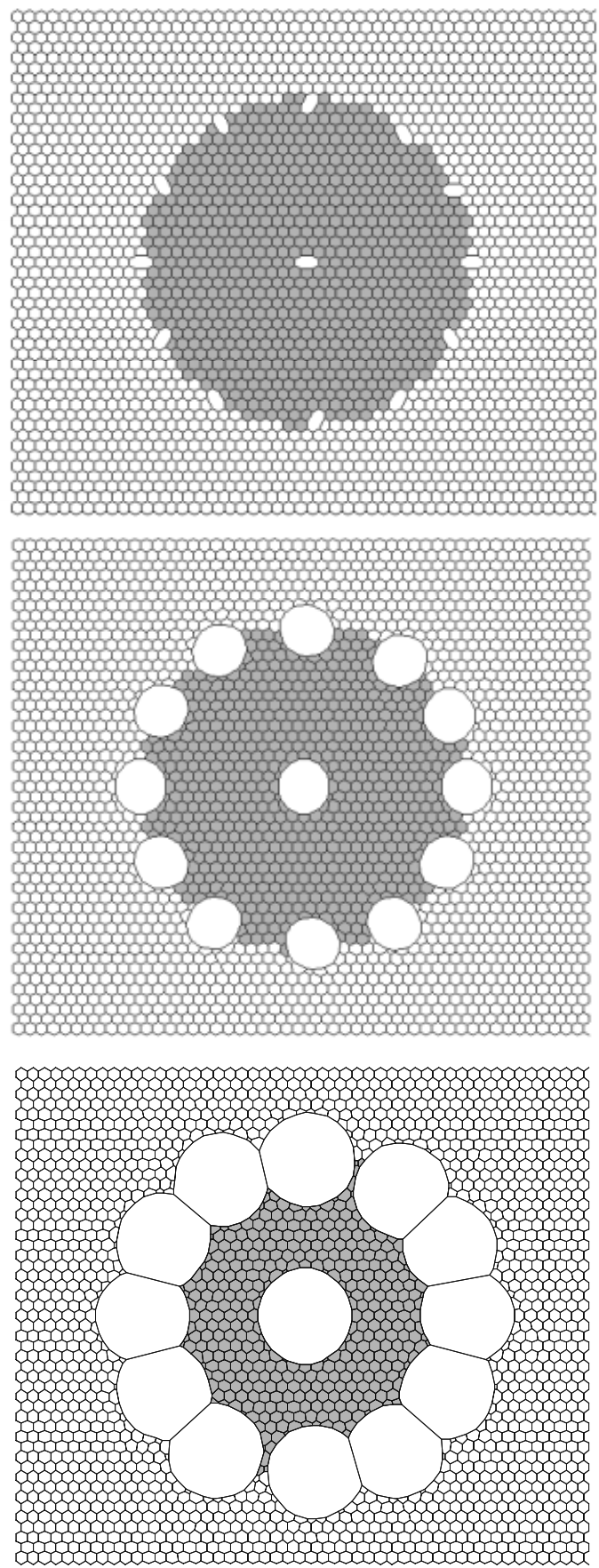

Fig. 2. Evolutionary behaviour of hexagonal froth, (system size $=2500$ ) with ring defects, after time steps of 100, 200, 240 


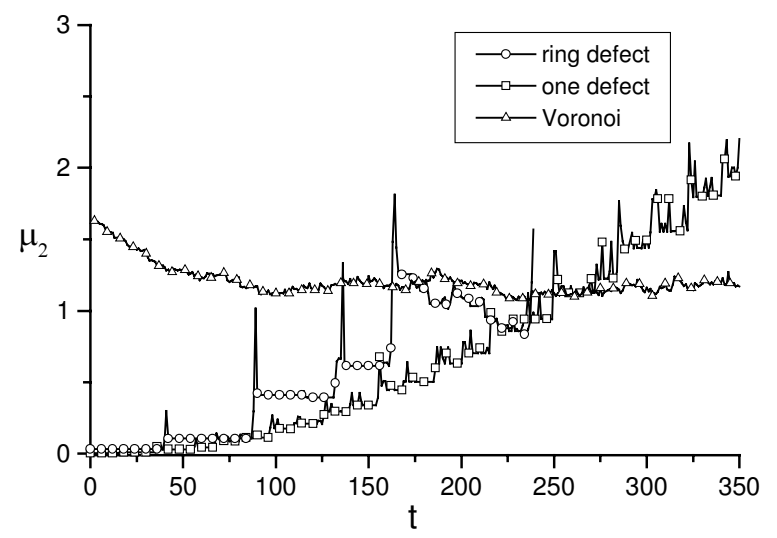

Fig. 3. Second moment, $\mu_{2}$ vs $t$ for ring defects, Voronoi and one defect case, (system size $=2500$ )

are consumed rapidly by the expanding central defect, whereas the trough in $k$ vs $\phi$ is both shifted in location, (to higher $\phi$ ) and less marked, as the ring expansion competes with that of the central defect. The convergence of the slope values is emphasised by the comparison with the one and several defects case, where $k$ varies little and is virtually constant in the former case. $\phi \rightarrow 1.0$, persistent fraction $\rightarrow$ constant value $=0$, in all cases, as noted also for the Voronoi, [19] .

\section{Discussion}

The virtual phase dynamics in the artificial froth system can be shown to vary enormously for specific colouring and values of $\phi$. Clearly, we can bias the evolution to permit survival of few or many of the coloured bubbles. Further, for any complex geometry in a moderate sized system, finite size effects must operate for enclosed regions of the froth and limit the evolution period. Persistent measurements based on ring geometry have shown similar behaviour to that obtained in both hexagonal and Voronoi network. Obviously, increasing the radius of the ring buffer leads to less extreme values of $k$, so that for very large radius, we are essentially back to the single defect case. Conversely, containment of the virtual phase by the defect rings acts as a buffer to rapid disappearance of cells and mimics slow decay of the persistent area fraction for increasing average area, (Fig. 5). If $<50 \%$ of the defects in the ring are coloured, little change is observed in the area ratio or persistent fraction as the large defect has major contribution to those properties. However, as the percentage increases further, large area ratio contributions do occur for small $\phi$. It seems clear that no persistent decay of the virtual phase may be observed for these systems, except for specific colouring patterns allied to the ring geometry. For all configurations of the virtual phase 

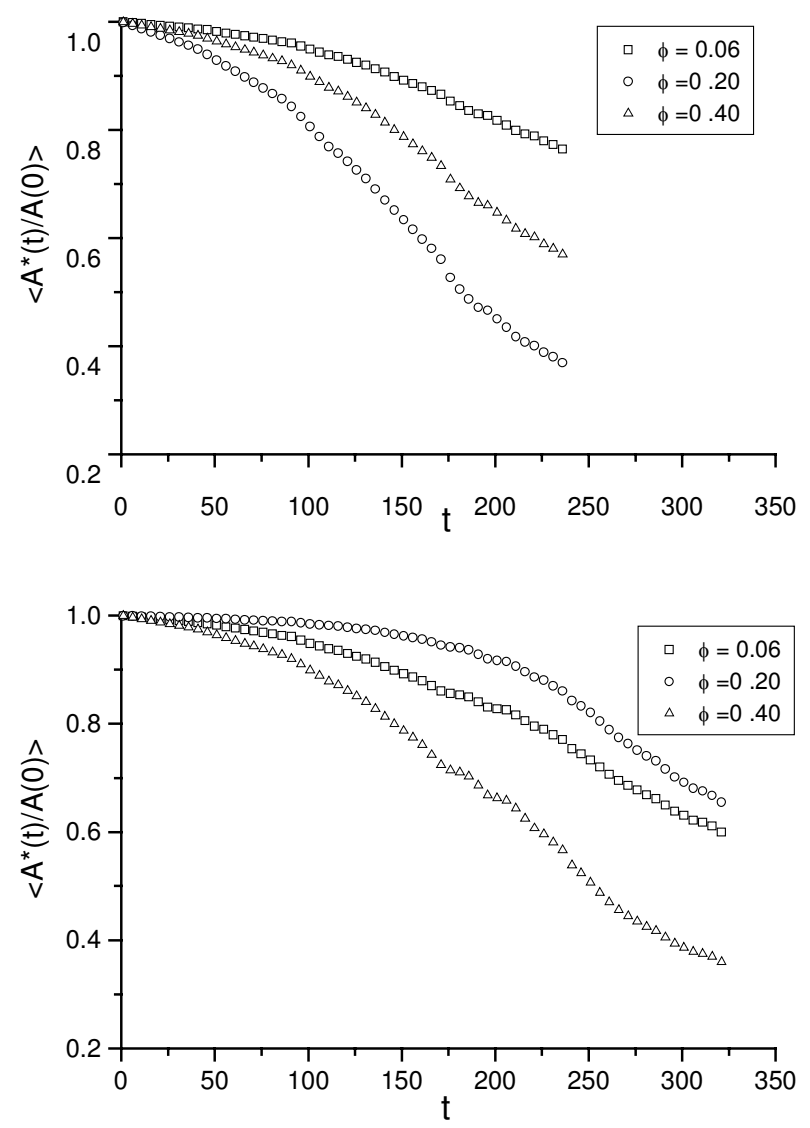

Fig. 4. Persistent area ratio, $\left\langle A(t)^{*} / A(0)\right\rangle$ (average area of persistent region) vs $t$, (time steps) for ring defect froth, with $\phi=0.06,0.2$ and 0.4. (a) for small ring, (b) for large ring.

and defect geometry, the limiting value, $k=0$, is approached gradually from below as $\phi \rightarrow 1.0$. Extreme values are due to the artificial constraints imposed on the froth evolution and are moderated as these are relaxed. For the constrained system, with the virtual phase contained by the defect ring, clearly

$$
k_{\max } \propto 1 / r
$$

Similar behaviour is observed for a ring geometry with fewer defects and the evolution can be monitored for a slightly longer period. However, time-scales in general are unacceptably short, since the closure of the ring effectively blocks further evolution, and it is evident that true persistence is not observed for the whole system. However, if we consider the ring geometries as a block between 

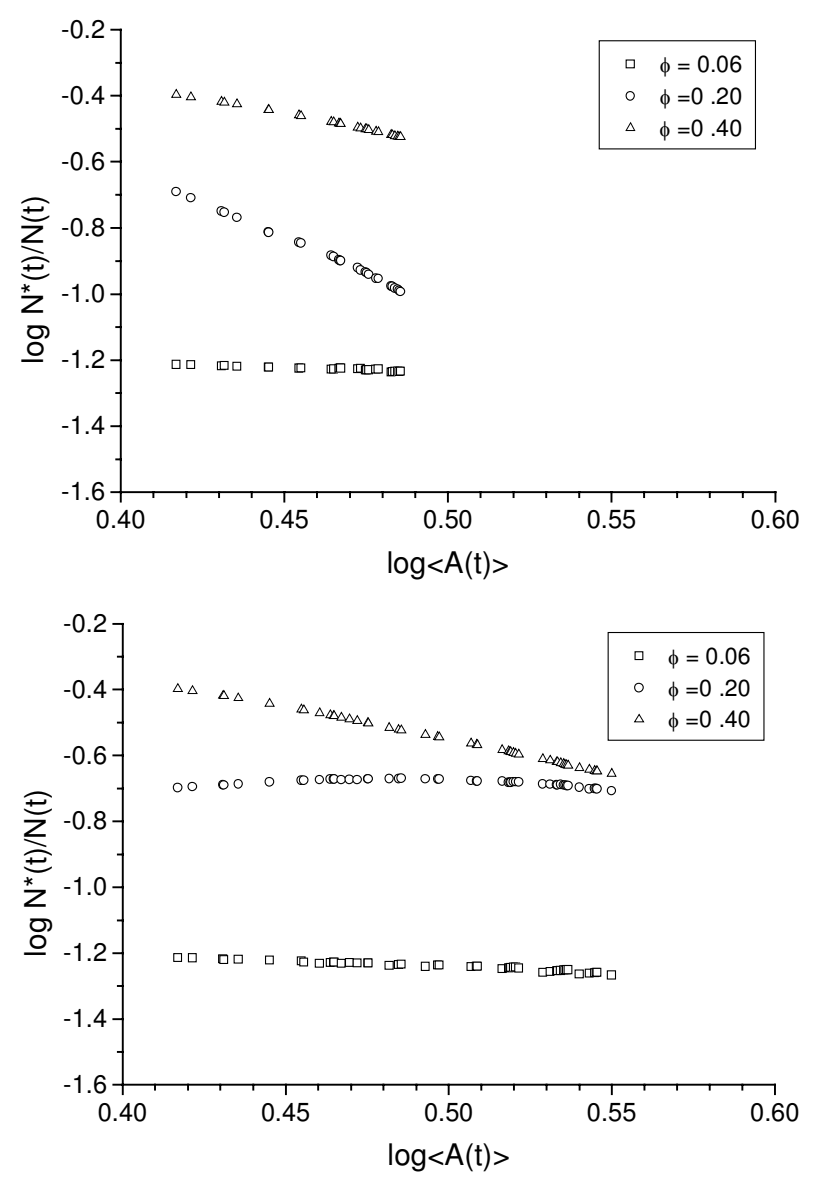

Fig. 5. Log.-log. plot of persistent fraction of bubbles, $N^{*}(t) / N(t)$ vs. average bubble area for ring defects froth with $\phi=0.06,0.2$ and 0.4. (a) and ( as in Fig. 4.

the inner and outer froth system, then depending on ring radius, contributions from those zones (in analogy with shell structure analysis) could bring us some new insights on virtual phase dynamics as well as other properties [24].

\section{Conclusions}

Construction of ring geometries in a 2-D froth has enabled further study of evolutionary properties and virtual phase dynamics. Evolution of the froth can be controlled by constrained geometries to some extent, with $\mu_{2}$ relatively stable at an earlier stage, which is consistent with a uniform froth with defects. However, true persistence has not been convincingly observed over these simulation times on a froth with ring defects, although various quantities, (the average area ratio 


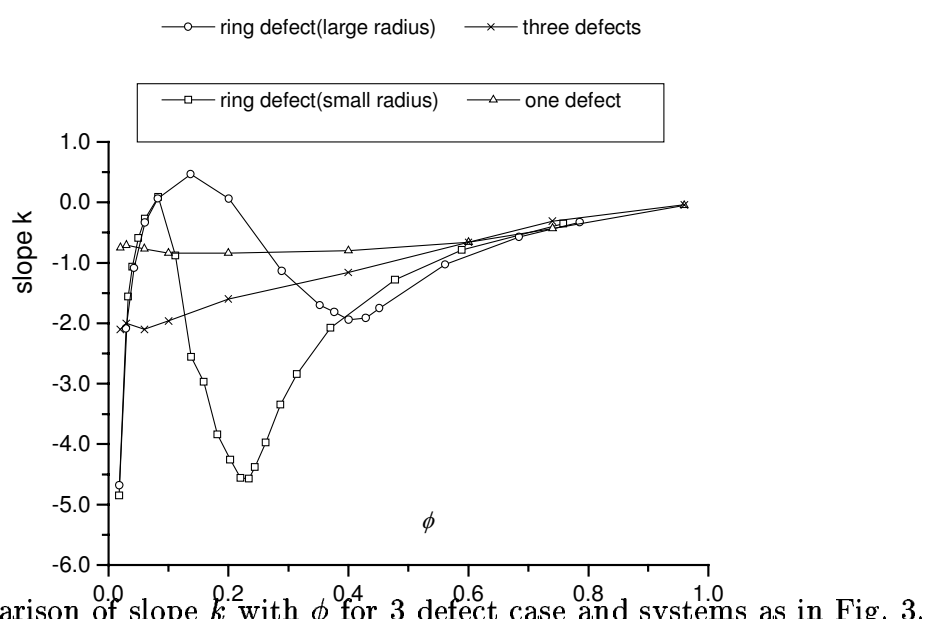

Fig. 6. Comparison of slope $k^{2}$ with $\phi^{0}$ for 3 defect case and systems as in Fig. 3 .

and fraction of coloured bubbles), have exhibited radically different behaviour for different values of $\phi$. These results are in line with those from defect-seeded or randomly defect-seeded hexagonal systems; in particular, it seems that the virtual phase, (as a persistence quantity), is contained by the defect ring buffer, with mimicked decays $\left(\log N^{*}(t) / N(t)\right.$ vs $\left.\log \langle A(t)\rangle\right)$ having non-zero slope $k$. It is evident that the non-zero decay exponent, a consequence of the artificial geometry, will tend to $\phi \rightarrow 1.0$ as $k \rightarrow 0$, in agreement with earlier findings for a Voronoi-based froth.

\section{References}

1. Weaire, D., Rivier, N.: Soap, cells and statistics - random patterns in two dimensions Contemp. Phys. 25 (1984) 59-99

2. Glazier, J.A., Gross, S.P., Stavans, J.: Dynamics of two-dimensional soap froths Phys. Rev. A 36 (1989) 306-312

3. Stavans, J.: The evolution of cellular structures Rep. Prog. in Phys. 56 (1993) 733789

4. Ruskin, H.J., Feng, Y: Cellular networks: characterising disorder in froths Trends in Stat. Phys. 2 (1998) 131-152

5. von Neumann J.: Grain shapes and other metallurgical applications of topology Metal Interfaces 41 (1952) 108-110

6. Levitan, B.: Evolution of two-dimensional soap froth with a single defect Phys. Rev. Lett. 72 (1994) 4057-4061

7. Ruskin H.J., Feng, Y.: Scaling properties for ordered/disordered 2-D dry froths Physica A 230 (1996) 455-466

8. Ruskin, H.J., Feng, Y.: A note on stages of evolution in a 2D froth Physica A 247 (1997) 153-158

9. Ruskin, H.J., Feng, Y.: The evolution of a two-dimensional soap froth with a single defect J. Phys.: Condens. Matter 7 (1995) L553-L559 
10. Aboav, D.A.: The arrangement of grains in a polycrystal Metallography 3 (1970) 383-390

11. Aboav, D.A.: The arrangement of cells in a net Metallography 13 (1980) 43-58

12. Weaire, D.: Some remarks on the arrangement of grains in a polycrystal Metallography 7 (1974) $157-160$

13. Szeto, K.Y., Aste, T., Tam, W.Y.: Topological correlations in soap froths Phys. Rev. E 58 (1998) 2656-2659

14. Dubertret, B., Rivier, N., Peshkin, M.A.: Long-range geometrical correlations in two-dimensional foams J. Phys. A: Math. Gen. 31 (1998) 879-900

15. Feng, Y., Ruskin, H.J.: Shell Analysis and effective disorder in a 2D froth. J. Stat. Phys. 99 (2000) 263-272

16. Bray, A.J., Derrida, B., Godreche, C.: Non-trivial algebraic decay in a soluble model of coarsening Europhys. Lett. 27 (1994) 175-180

17. Tam, W.Y., Rutenberg, A.D., Szeto, K.Y., Vollmayr-Lee, B.P.: Cluster persistence: A discriminating probe of soap froth dynamics Europhys. Lett. 51 (2000) 223-229

18. Majumdar, S.N.: Persistence in nonequilibrium systems Curr. Sci. 77 (1999) 370375

19. Ruskin, H.J., Feng Y., Zhu, B.: Modelling froth dynamics; persistence measures Physica A 293 (2001) 315-323; also Ruskin, H.J., and Feng Y.: Constrained geometries in soap froth dynamics, at conference on Horizons in Complex Systems, Messina, Sicily, Italy, Dec 5th-8th, 2001

20. Aste, T., Boose, D., Rivier, N.: From one cell to the whole froth: a dynamical map Phys. Rev. E. 53 (1996) 6181-91.

21. Weaire, D., Kermode, J.P.: Computer simulation of a two-dimensional soap froth Phil. Mag. B 48 (1983)245-259; 1984. Phil. Mag. B 50 (1984) 379-395

22. Kermode, J.P., Weaire, D.: 2D-FROTH: a program for the investigation of 2dimensional froths Comp. Phys. Commun. 60 (1990) 75-109

23. Tam, W.Y., Zeitak, R., Szeto, K.Y., Stavans, J.: First-passage exponent in twodimensional soap froth Phys. Rev. Lett. 78 (1997) 1588-1591

24. Feng, Y., Ruskin, H.J., Zhu, B.: 2002 (in preparation) 